\title{
Gastrointestinal Mantle Cell Lymphoma: incidental finding on routine CT scan following pneumothorax.
}

\author{
Mingma Thsering Sherpa, ${ }^{a^{*}}$ Damiano Rondelli, ${ }^{a}$ Raksha Shrestha ${ }^{\mathrm{a}}$
}

${ }^{a}$ Section of Hematology/Oncology, University of Illinois Hospital and Health Science System and University of Illinois Cancer Center, Chicago, IL, USA

\author{
Accepted on \\ December 20 2012 \\ DOI Name \\ 10.3126/jaim.v2i1.7635

\section{Keywords} \\ Diagnosis, gastric mantle cell lymphoma, gastric \\ mass, treatment. \\ Citation \\ Sherpa MT, Rondelli D, Shrestha R. Gastrointestinal \\ Mantle Cell Lymphoma: incidental finding on \\ routine CT scan following pneumothorax. Journal \\ of Advances in Internal Medicine 2013;02(01):24-6
}

\begin{abstract}
Gastric mantle cell lymphoma is a rare form of gastrointestinal tumor and represents 2.5-7\% of all non-Hodgkin's lymphomas. A 69-year-old maleadmitted with spontaneous pneumothorax was found to have a gastric masson CT scan. Results of histopathological,immunohistochemical and genetic analysis were consistent with mantle cell lymphoma(MCL).Patient responded well to intensive chemotherapy and subsequent allogeneic hematopoietic stem cell transplant and remains in complete remission. We would like to emphasize that a gastric mass, as in this case can occur as a rare presentation of MCL involving the gastrointestinal tract without any other overt signs and symptoms.
\end{abstract}

\section{INTRODUCTION}

Mantle cell lymphoma ( $\mathrm{MCL}$ ) is a distinct subtype of B-cell lymphoma accounting approximately 3-10\% of lymphoma diagnoses. ${ }^{1}$ About $2.5-7 \%$ of all Non-Hodgkin lymphoma cases are mantle cell and despite being a low-grade lymphoma, it has the worst prognosis among all lymphomas. It tends to occur in older adults with a median age of approximately 60 years and is common in males (M: F-2-7:1). 2,3 Primary gastric mantle cell lymphoma is an extremely rare form of gastrointestinal tumor and represents $2.5-7 \%$ of all non-Hodgkin's lymphomas. ${ }^{4}$ In the present report, we describe the clinicopathologic features of a case of early primary gastrointestinal mantle cell lymphoma found incidentally on routine Computed Tomography (CT) scan following pneumothorax.

\section{CASE REPORT}

A 69-year-old male with a past medical history of gastroesophageal reflux disease, atrial flutter, hypertension and anxiety presented in the emergency department with sudden chest pain and shortness of breath and was diagnosed with spontaneous pneumothorax. Physical examination did not reveal any palpable peripheral lymph nodes, liver or spleen enlargement or any pathologic abdominal mass. His Computed Tomography (CT) scan of the chest revealed a small right pneumothorax with subcutaneous emphysema and a $3 \mathrm{~cm}$ gastric fundal mass with irregular thickening of gastric wall. Numerous mildly enlarged paragastric lymph nodes were also noted. He underwent an esophagogastroduodenoscopy that revealed a normal esophagus and several gastric masses with two (large greater than $3 \mathrm{~cm}$ ) in the fundus and a few satellite lesions in the antrum with thickened folds. Positron Emission Tomography (PET) /CT showed large hypermetabolicexophytic soft tissue mass from gastric antrum, intense hypermetabolic area in the cecum/ileocecal valve with multiple enlarged mesenteric lymph nodes. Multiple biopsies taken showed lymphoma cells positive for CD20, CD5, BCL2 and negative for CD10 and CD 23. Initial diagnosis of mantle cell lymphoma involving intra-abdominal lymph nodes was made (ICD9 200.43, working diagnosis). His LDH was normal and $\beta 2$ microglobulin was mildly elevated at 2.53. Bone marrow evaluation revealed hypocellularity(20-25\% cellular) with subcortical trephine core biopsy showing multiple crushed lymphoid aggregates. Marrow-aspirate smears showed approximately $13 \%$ lymphocytes with occasional small atypical forms, erythroid hyperplasia and adequate granulopoiesis and megakaryopoiesis without significant dyspoiesis or increased blasts (1\%). Immunohistochemical stains performed on core biopsy showed the lymphoid aggregates to be predominantly CD20 and CD79a positive B-cells with aberrant CD\% expression occupying approximately 10$15 \%$ subcortical marrow space. Cyclin D1 (BCL-1) was negative within the neoplastic lymphocytes. KI-67 showed a proliferative activity of approximately 5-10\%. Flow cytometry analysis of marrow aspirate showed CD5 positive lambda-monoclonal B cell population. Fluorescent In Situ Hybridization (FISH) study was positive for CCND1 (BCL1) rearrangement. Karyotypic studies showed a normal male karyotype. Marginal anemia, slight absolute neutrophilia without left-shift with no lymphocytosis was seen on peripheral blood smear. Reported findings were consistent with approximately $10-15 \%$ marrow involvement by mantle cell lymphoma.

Patient was started on an intensive chemotherapy with hyper-Cytoxan, Vincristin, Doxorubicin (Adriamycin), Dexamethasone (CVAD) andrituxan. He completed cycle\#1 (part A and part B) and cycle\#2 (part A and part B) along with Neulasta (Pegfilgastrim) support. He then underwent a successful peripheral blood stem cell mobilization (with Neupogen) and

\footnotetext{
* Corresponding author

Dr. Mingma Thsering Sherpa

Clinical Elective Student

Section of Hematology/Oncology,

University of Illinois Hospital and Health Science System and University of Illinois Cancer Center, Chicago, IL, USA

Email: mingma.thsering@gmail.com
} 
was then admitted for autologous stem cell transplantation during which hereceived BEAM-rituxan conditioning. He engrafted neutrophils and platelets. He had hypogammaglobulinemia secondary to rituxan and chemotherapy for which he received IVIG. Pentamidineprophylaxis was given for pneumocystis carinii pneumonia (PCP).

He subsequently recovered and afollow up PET/CT showed no evidence of lymphoma and his bone marrow biopsy was negative for residual or recurrent disease.

\section{DISCUSSION}

The genetic hallmark of mantle cell lymphoma is the chromosomal translocation $t(11 ; 14)(q 13 ; q 32)$ which leads to the deregulation and upregulation of Cyclin D1 which is an important regulator of the G1 phase of the cell cycle. ${ }^{5}$ Cyclin D1 plays an important role in the cell cycle regulation G1-S transition following mitotic growth factor signaling. Cyclin D1 binds to CDK4 and CDK6 to form a CDK/cyclin complex able to phosphorylate the tumor suppressor retinoblastoma (RB1) facilitating cell cycle progression. Thus, cyclin D1 overexpression would contribute to the lymphomagenesis in $\mathrm{MCL}$ by overcoming the suppressor effect that retinoblastoma performs in the $\mathrm{G} 1 / \mathrm{S}$ transition. ${ }^{3}$

Majority of patients with mantle cell lymphoma present with advanced stage disease $(70 \%)$. While $75 \%$ of the patients initially present with lymphadenopathy, extranodal disease is the primary presentation in the remaining $25 \% .{ }^{6}$ Common sites of nodal involvement are the lymph nodes, spleen (45-60 percent), Waldeyer's ring, bone marrow (>60 percent), blood (13-77 percent) and extranodal sites like the gastrointestinal tract. ${ }^{7,8}$ Mantle cell lymphoma can involve any region of the gastrointestinal tract, occasionally presenting as multiple intestinal polyposis. A prospective study on gastrointestinal involvement of mantle cell lymphoma showed the following results: stomach (57 percent), duodenum (52 percent), jejunum/ileum (87 percent), colon (90 percent) and rectum (69 percent). ${ }^{9}$ Up to a third of patients have systemic B symptoms, such as fever, weight loss and night sweats.

Initial diagnostic evaluation include a complete blood count, blood chemistries for renal and liver function; computed tomography scans of chest, abdomen and pelvis and an aspiration and biopsy of the bone marrow. Many patients have circulating lymphoma cells detectable by a peripheral blood smear or by flow cytometry. ${ }^{10,11}$ Immunohistochemistry can still be false negative, mainly due to the quality of the material. ${ }^{12}$

Cell proliferation (Ki-67) has been exploratively analyzed as an important biologic marker and has showed strong additional prognostic relevance. CCND1 expression, due to the presence of $\mathrm{t}(11 ; 14)(\mathrm{q} 13 ; \mathrm{q} 32)$ translocation, is present in virtually all cases of $\mathrm{MCL}^{12}$ however, a very few cases that do not express CCND1 might exist. ${ }^{13}$ Recently a new prognostic model has been introduced by the German Lymphoma Study Group called the MIPI (MCL International Prognostic Index) on the basis of age, performance status, serum lactate dehydrogenase and leukocyte count. ${ }^{14}$ As per MIPI,

\section{REFERENCES}

1. Sander B. Mantle cell lymphoma: recent insights into pathogenesis, clinical variability, and new diagnostic markers. Semin Diagn Pathol 2011;28:245-55.

2. Andersen NS, Jensen MK, de Nully Brown P, et al. A Danish population based analysis of 105 mantle cell lymphoma patients: patients are classified into low risk (44\% of patients, median overall survival not reached), intermediate risk (35\%, 51 months), and high-risk groups (21\% 29 months).

The treatment approach to newly diagnosed patients with $\mathrm{MCL}$ depends on the patient's eligibility for stem cell transplantation (SCT). Those who are eligible are usually treated with either rituximab-CHOP (cyclophosphamide, doxorubicin, vincristine and prednisone) followed by SCT or rituximab-HyperCVAD (cyclophosphamide, vincristine, doxorubicin, decadron, cytarabine, and methotrexate) followed by observation. ${ }^{15}$

There are basically three potential induction regimens among several: 1) Standard doxorubicin-containing regimens such as R-CHOP 2) intensive combination chemotherapy regimens, including anti-metabolites such as R-HyperCVAD; and 3) purine analog-based regimens such as R-FCM (fludarabine, cyclophosphamide, and mitoxantrone).There have been limited studies using single-agent radioimmunotherapy (RIT) for patients with relapsed $\mathrm{MCL}$.

The role of HSCT remains controversial and is not clearly defined despite the availability of newer therapies in MCL. Most of the studies that analyzed the role of $\mathrm{HSCT}$ in $\mathrm{MCL}$ were single-center trials with small number of subjects. Thus individualizing therapeutic approaches are essential to improve outcome in this aggressive disease. ${ }^{16}$ If the patient is $<75$ years old and in excellent physical condition, then autologous stem cell transplantation (SCT) can be considered a potential option but if the patient is elderly and not a SCT candidate, the use of rituximab-containing chemotherapy regimen is appropriate. ${ }^{15}$

Newer agents have been identified for relapsed MCL. Proteosome inhibitors like Bortezomib are inhibitors of the intracellular protein degradation pathway known as the proteosome and has shown antitumor activity in relapsed $\mathrm{MCL}$ in several studies. Thalidomide, used primarily for multiple myeloma is another agent that has been evaluated in $\mathrm{MCL}$ alone and in combination. ${ }^{15}$ Temsirolimus is an inhibitor of the mammalian target of rapamycin (mTOR) targeting the phospatidyl inositol 3 kinase pathway. ${ }^{17}$ This mechanism inhibits the translation of mRNA, including cyclin D1 mRNA, making this agent a sound therapeutic agent in MCL.

\section{CONCLUSION}

MCL has the most aggressive clinical course thus necessitating the need for early diagnosis and management. Patients generally present at later stages of disease with extensive involvement of lymph nodes. Nevertheless, any gastric mass, as in this case can be a rare presentation of primary isolated gastrointestinal MCL without any other overt signs and symptoms.Cyclin D1 plays an important role in pathogenesis of $\mathrm{MCL}$ and is a highly specific marker for diagnosis.No standard guidelines have been established till date for the treatment of $\mathrm{MCL}$ and its prognosis remains poor. Our patient responded well to intensive chemotherapy and subsequent allogeneic hematopoietic stem cell transplantand remains in complete remission.

Incidences, clincal features, response, survival and prognostic factors. Eur J Cancer 2002;38:401-8.

3. Jares $P$, Campo $E$. Advances in the understanding of mantle cell lymphoma. Br J Haematol 2008;142:149-65.

4. Chong $\mathrm{Y}$, Shinn JJ, Cho MY, et al. Synchronous primary gastric mantle cell lymphoma and early gastric carcinoma: a case report. Pathol Res Pract 2008;204:407-11. 
5. Fernandez V, Hartmann E, Ott G, et al. Pathogenesis of MantleCell Lymphoma: All Oncogenic Roads Lead to Dysregulation of Cell Cycle and DNA Damage Response Pathways. J Clin Onc 2005;23:6364-9.

6. Argatoff LH, Connors JM, Klasa RJ, et al. Mantle cell lymphoma: a clinicopathologic study of 80 cases. Blood 1997;89:2067-78.

7. Romaguera JE, Medeiros LJ, Hagemeister FB, et al. Frequency of gastrointestinal involvement and its clinical significance in mantle cell lymphoma. Cancer 2003;97:586-91.

8. Ferrer A, Salaverria I, Bosch F. Leukemic involvement is a common feature in mantle cell lymphoma. Cancer 2007;109:2473-80.

9. Ruskone-Fourmestraux A, Delmer A, Lavergne A. Multiple lymphomatous polyposis of the gastrointestinal tract: prospective clinicopathologic study of 31 cases. Groupe D'étude des Lymphomes Digestifs. Gastroenterology 1997;112:7-16.

10. Hanson CA, Kurtin PJ, Katzmann JA, et al. Immunophenotypic analysis of peripheral blood and bone marrow in the staging of B-cell malignant lymphoma. Blood 1999;94:3889-96.
11. Cohen PL, Kurtin PJ, Donovan KA, et al. Bone marrow and peripheral blood involement in mantle cell lymphoma. $\mathrm{Br} J$ Haematol 1998;101:302-10.

12. Bertoni F, Rinaldi A, Zucca E, et al. Update on the molecular biology of mantle cell lymphoma. Hematol Oncol 2006;24:22-7.

13. Fu K, Weisenburger DD, Greiner TC, et al. Cyclin D1-negative mantle cell lymphoma: a clinicopathological study based on gene expressing profiling. Blood 2005;106:4315-21.

14. Hoster E, Dreyling M, Klapper W, et al. A new prognostic index (MIPI) for patients with advanced-stage mantle cell lymphoma. Blood 2008;111:558-65.

15. Witzig TE. Current treatment approaches for mantle-cell lymphoma. J Clin Oncol 2005;23:6409-14.

16. Ghielmini M, Zucca E. How I treat mantle cell lymphoma. Blood 2009;114:1469-76.

17. Rini BI. Temsirolimus, an inhibitor of mammalian target of rapamycin. Clin Cancer Res 2008;14:1286-90. 